\title{
A novel technique for hypersonic vehicle control
}

\author{
Dimitrios Mitridis ${ }^{1}$, Chris Bliamis ${ }^{1}$, Pericles Panagiotou ${ }^{1}$, and Kyros Yakinthos ${ }^{1,2, *}$ \\ ${ }^{1}$ UAV integrated Research Center (UAV-iRC), Center for Interdisciplinary Research and Innovation \\ (CIRI), Aristotle University of Thessaloniki, 57001, Thessaloniki, Greece \\ ${ }^{2}$ Laboratory of Fluid Mechanics and Turbomachinery, Department of Mechanical Engineering, \\ Aristotle University of Thessaloniki, 54124, Thessaloniki, Greece
}

\begin{abstract}
A novel control technique is investigated for hypersonic aerial vehicles. The technique is based on the use of active shock bumps (SBs) as a form of control device. The SBs deflect to create shockwaves on-demand, at specific locations around the aerial vehicle. As a result, a force is applied on the aerial vehicle, which in turn is used to provide the necessary moment for pitch and roll manoeuvres. In this work, a preliminary aerodynamic analysis of the SB device technique is made by means of CFD. For this purpose, and taking the large corresponding Reynolds numbers of the flow into consideration, the two-dimensional Euler equations are solved. A parametric investigation is carried out, by examining the effect of key parameters, namely the Mach number $(\mathrm{M})$ and device deflection angle $\left(\delta_{\mathrm{SB}}\right)$ on the produced force acting on the vehicle, serving as a proof of concept. Using a specific interpolation method, the resultant force is presented as a function of the Mach number and the device deflection angle, on threedimensional charts, where the effect of each parameter is shown (forceMach-deflection maps). Furthermore, a preliminary feasibility study is performed, including a kinematic analysis and some key material considerations. Additionally, a kinetic analysis is also conducted to secure the dynamic rigidity of the actuating mechanism and provide an initial estimation concerning weight and basic geometrical parameters of the SB mechanism components.
\end{abstract}

\section{Introduction}

A prominent issue in conventional control surfaces and stabilizers, used in most aerial vehicles, is the presence of shockwaves, which limit their effectiveness and can lead to loss of control, or even control reversal [1,2]. Designers usually oversize the control surface or select all-moving ones, or both [3]. This solution adds a considerable drag penalty on the aerial vehicle, due to the increased trim and friction drag (because of the increased wetted area), regardless if they are operating or not. In this paper, an alternative solution is proposed utilizing Shock Control Bumps (SBs) that can be used for aircraft control, on aerial vehicles operating in the higher supersonic and hypersonic regime, such as the Boeing X-51 [4] and the SR-72 UAV concept [5].

\footnotetext{
* Corresponding author: kyak@auth.gr
} 
Conventional shock control bumps have been widely investigated in the past decades as a means of reducing wave drag [6,7]. However, in an alternate approach, the SBs can be used to create shockwaves on demand, which alter the velocity and pressure fields in desired locations around the aerial vehicle, thus producing a force which can provide the necessary moment for pitch, roll and yaw maneuvers, as shown in Fig. 1.

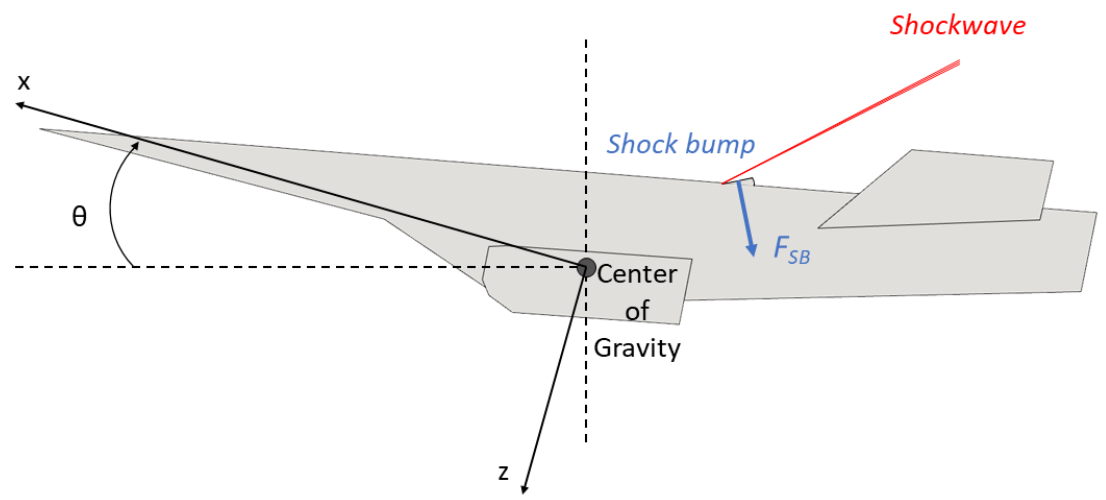

Fig. 1. Conceptual drawing showing the philosophy of the SB control device on a reference hypersonic vehicle [8].

The proposed technique is investigated both from an aerodynamic and a kinematic perspective at TRL1. Concerning aerodynamics, a parametric aerodynamic analysis is performed by means of CFD, solving the Euler equations in order to have a first assessment of the concept at TRL1. A synergetic approach is employed, where the CFD results provide the input for the pressure loads applied on the mechanism. After completing the conceptual calculations regarding the operation mechanism of the SB, a CAD model is constructed followed by a kinematic and kinetic analysis. The trajectory and the velocity of the mechanism are calculated, as are the forces developed on the mechanism bars.

\section{Tools and Methods}

\subsection{Parametric investigations}

The examined SB device is presented in Fig. 2. The shock bump is represented as a 2dimensional wedge with its rear side (shape) being an arc of a circle, whose center is located at the apex of the SB. Based on preliminary calculations, the length of the device $\left(1_{\mathrm{SB}}\right)$ is set to $20 \mathrm{~cm}$ for all cases, while the deflection angle $\left(\delta_{\mathrm{SB}}\right)$ is variable (Fig. 2). More specifically, the examined $\delta_{\mathrm{SB}}$ ranges between 0 and $40 \mathrm{deg}$, at steps of $10 \mathrm{deg}$. Regarding the freestream velocity, flight Mach numbers of 4, 5, 6 and 7 are selected, to ensure that an adequately high force for control purposes is generated. The ambient conditions correspond at a flight altitude of 70.000ft [9], selected based on the Lockheed SR-71 operating altitude.

\subsection{CFD methodology and validation}

A preliminary aerodynamic analysis is performed by means of CFD, for the evaluation of the $\mathrm{SB}$ device. The flow is considered two-dimensional so that the complexity of threedimensional phenomena is neglected. Furthermore, the flow is regarded inviscid, since the Reynolds number excides $10^{6}$ for the selected freestream Mach number range (Mach 4 to 7 ) $[10,11]$. Those simplifications are deemed sufficient to capture the main aspects of the flow, i.e. the shockwave location and strength, as well as determine the pressure distribution on the SB device and the flowfield downstream [12]. Therefore, the two-dimensional Euler equations are solved, using the commercial ANSYS CFX software (Release 18.2). 


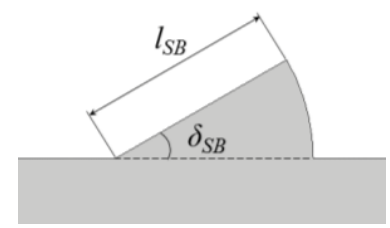

Fig. 2. Drawing of the proposed SB device, where the key geometric characteristics are presented.

To select the optimum number of nodes for the aerodynamic investigation, a grid dependency study is conducted at Mach 5 and a $\delta_{\mathrm{SB}}$ of $30 \mathrm{deg}$. Based on this study, a grid of 0.8 million nodes is eventually selected for the rest of the parametric investigations [13].

A two-stage validation of the CFD methodology is performed [13], on a simple geometry, and on the actual SB respectively. Initially, the flowfield of a two-dimensional wedge of angle $\theta$ equal to $20 \mathrm{deg}$, with a freestream velocity corresponding at Mach 5 is computed. The shockwave angle $\beta$ is found to be $30 \mathrm{deg}$, matching the theoretical predictions [14]. Then, a further validation for the CFD methodology is performed using the Mach independence principle [15]. Specifically, the pressure coefficient $\left(\mathrm{C}_{\mathrm{P}}\right)$ on the SB device (Fig. 2 ) in respect to different velocities is examined, and was found to be practically independent of the Mach number in the hypersonic regime (i.e. Mach number greater than 5), in complete agreement with the Mach independence principle governing inviscid hypersonic flows.

\subsection{Kinematic methodology}

To introduce a feasibility check for this vehicle control method, a mechanical model has been conceptualized and solved for the actuating mechanism of the bump. To determine the concept's main working aspects, a geometrical solution of the mechanism was carried out. This, solution is able to provide the basic dimensions of the mechanism and the acceptable angles between its members. In addition, a CAD assembly was created, focusing on a dimensional overview of the mechanism, based on the conceptual calculations [16]. The actuating system is determined as a hydraulic piston system. The CAD assembly, presented in Fig. 3, is the testbed for creating a working kinematic model. The rear side of the SB, not shown here, will be consisted of a number of curved plate parts, in order to provide the desired geometry as shown in Fig. 2 and also as not to collide with the actuating hydraulic piston.

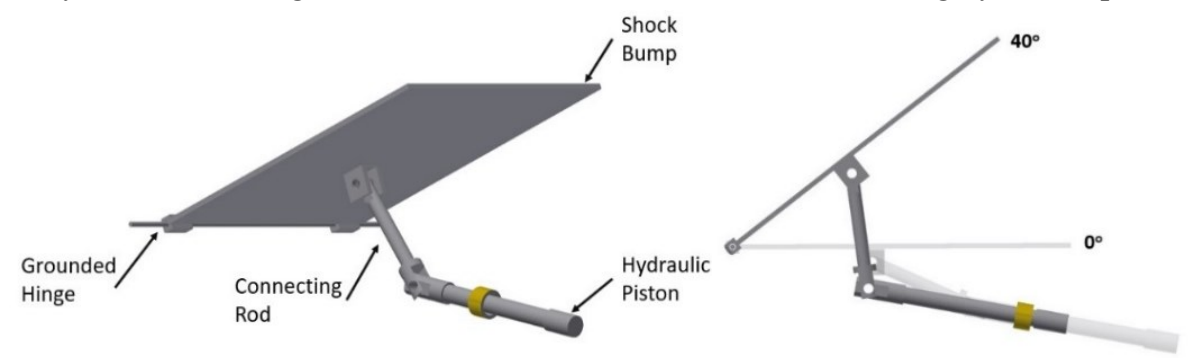

Fig. 3. SB mechanism system CAD model.

The conceptual CAD model consists of a hydraulic piston actuator, a connecting rod and the bump, ascending from the vehicle's skin. The piston is able to move in straight line between two, well selected, front and aft positions. The aft position of the piston forces the bump to be completely closed (parallel to the vehicle's skin) and the front position places the bump on a 40deg deflection angle. Concerning the bump itself, it is firmly anchored on a revolute joint, connecting it to the rest of the skin. As clarified in Fig. 3, the rod works as a medium, translating the linear actuating movement of the piston to a circular orbit of the bump, around its anchor point [16]. Because of the constrained bump's path and the limitations about the front and aft piston positions, the mechanism is designed as a non- 
singular one, which induces the absence of non-continuity positions. In other words, no position exists, where the mechanism can be kinetically "jammed" if its velocity is zero [16]. The non-singular mechanism is achieved by selecting a four-bar mechanism (ground included) with a limited deflection field from zero to $40 \mathrm{deg}$ for the last bar, i.e. the shock bump. The first bar of the mechanism is the actuating piston and the second one is the connecting rod. Note that the representation is limited on a conceptual level for the needs of this paper, i.e. the mechanism bars are designed as simple rods, and the bump as a simple flat surface. The detailed mechanism should be a more complex mechanical assembly, with back up rods in both sides of the bump, buffers at the end of the designed travel and many other features composing a fully operational system [16].

Regarding the kinematic model, the BETA ANSA 19.1.1 software is used, which gives the ability of a full kinematic and kinetic analysis of a mechanism during its design process. For the kinematic analysis it was decided that a 2D model would be sufficient enough giving realistic results about the forces evolving on the mechanism joints. The simulation time is set to be $0.1 \mathrm{sec}$ for a complete deployment, as a realistic time for a control system [2]. The external forces applied to the bump are provided by the CFD analysis and serve as an input to current model, in a one-way Fluid-Structure Interaction (FSI) approach. More specifically, a function relating the magnitude of the aerodynamic force with the deflection angle and the Mach number is generated. The piston force needed for the mechanism work is being calculated by the current kinetic analysis allowing an applicable design for the piston actuator. The forces being developed on every joint are also calculated, allowing for a realistic design of the mechanical connectors of the system.

An extended literature review regarding mechanisms on hypersonic applications, indicates a quite narrow range of materials used. The most common materials for these applications are titanium or aluminum alloys, carbon fibers, reinforced carbon-carbon, ceramics, titanium aluminide alloys and superalloys [17]. In this research, the material evaluation study was conducted on a conceptual level, aiming at an initial material family selection, considering their physical and mechanical properties. The study initiates with a desirable material property listing, suitable for hypersonic applications. Some of the most critical parameters (e.g. Yung's Modulus, density or creep temperature) are listed first, introducing a property hierarchy which facilitates an easier material family selection. Likewise, other non-critical material properties, such as cost, development level or machinability, are taken into account as well. The initial material family selection is followed by a secondary feasibility analysis, based on technical reviews regarding existing hypersonic vehicles [18]. The study concludes with the selection of a reference material, from the previously selected material family, as an input for physical and mechanical properties needed to calculate the dimensions and mass for this concept SB mechanism.

Given a concept SB with dimensions of $200 \mathrm{~mm}$ by $150 \mathrm{~mm}$, the mechanism weight estimation study is based on an initial sizing of the mechanism parts considering the maximum static loads developed during its operational phases. These loads are used as boundary conditions for a finite element analysis (FEA), through which structural integrity of the mechanism parts is verified for its working load range.

Concerning the mechanism rods, it is identified that the main loading condition is compression, given that all joints of the mechanism are frictionless. During compression the prospect of buckling, on every one of the two working rods, should be avoided, by applying stresses lesser than the maximum allowed by Euler's buckling equation. The maximum stress (Euler buckling stress) that a rod of certain diameter can withstand without buckling can be calculated analytically by the following equation.

$$
\sigma_{\text {Euler }}=\mathrm{F}_{\text {Euler }} / \mathrm{A}=\frac{k \pi^{2} E}{(L / r)^{2}}
$$


Ultimately, the combined kinematic and kinetic analysis results are used to fully define the geometrical and physical parameters of the mechanism's parts, their shape, their overall volume and density, and therefore their mass.

\section{Results}

The pressure coefficients $\mathrm{C}_{\mathrm{P}, \mathrm{SB}}$ for the intermediate Mach numbers and $\delta_{\mathrm{SB}}$ are interpolated from the data of the parametric investigations. The results of those interpolations are summed up in Fig. 4.

From Fig. 4 , it is clear that the $\delta_{\mathrm{SB}}$ has a strong and dominant effect on the pressure coefficient $\mathrm{C}_{\mathrm{P}, \mathrm{SB}}$, whereas the effect of the freestream Mach number is far smaller and almost negligible. This observation is in accordance with theoretical results, as dictated by the Mach independence principle [15].

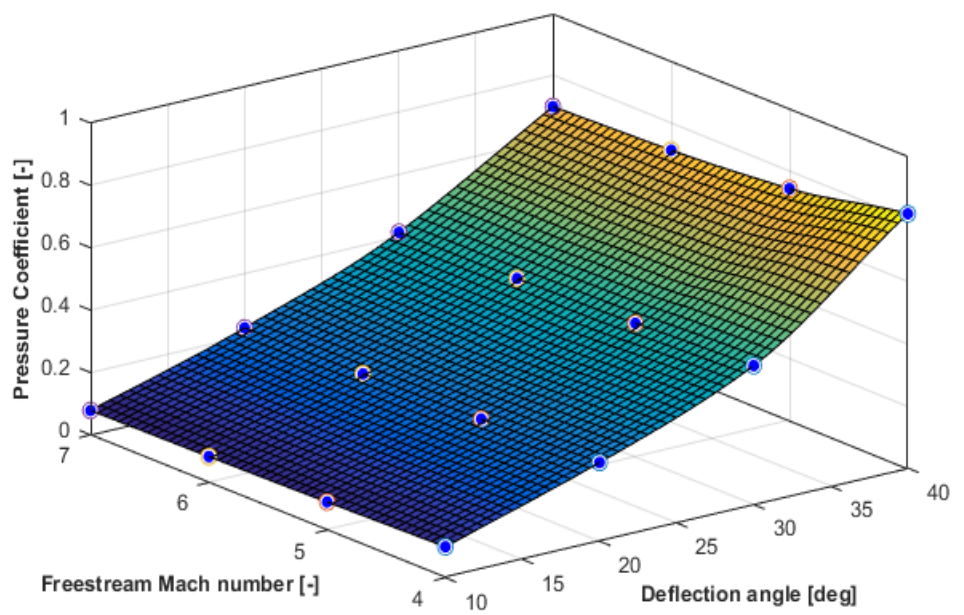

Fig. 4. Pressure coefficient on the SB, as a function of Mach number and device deflection $\delta_{\mathrm{SB}}$.

Concerning the kinematic results, the main diagram plots the $\delta_{\mathrm{SB}}$ with respect to the time of deployment $(0-0.1 \mathrm{sec})$, which tends to maintain a constant form, independent of Mach number (Fig. 5, Deflection Angle). The presented mechanism accelerates less on the first steps of deployment and follows a more linear behavior until complete deployment status. This tendency to a more linear behavior at highest, angles of deflection, is considered desirable, allowing the mechanism to cope more efficiently with the aerodynamic loads, ignoring the inertial loads of the mechanism as the current acceleration tends to zero.

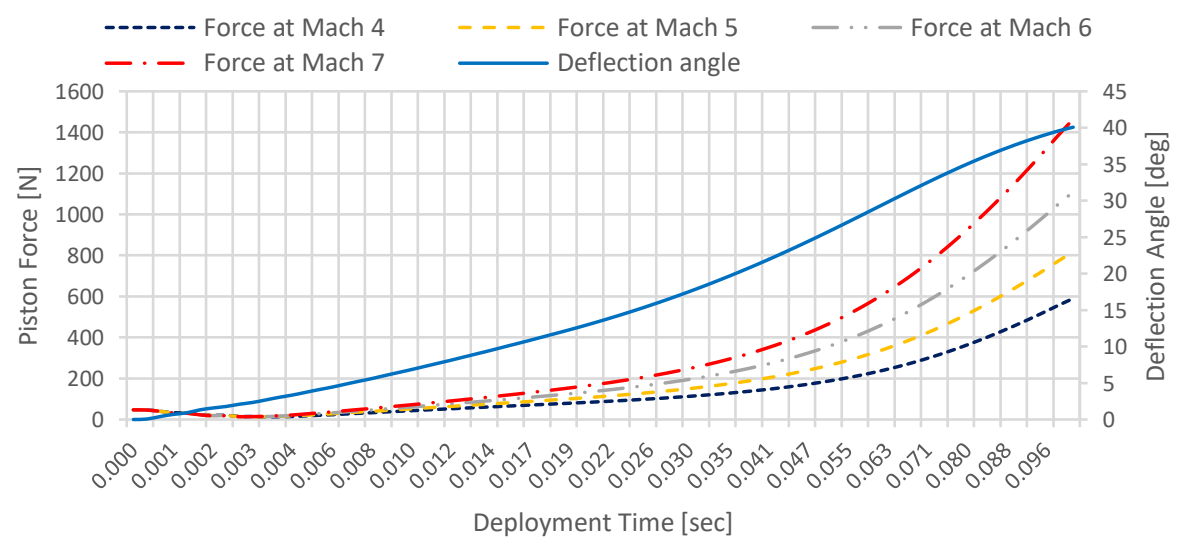

Fig. 5. Actuating piston force vs deployment time. 
Another crucial element of the current analysis is the forces required to actuate the whole mechanism. In Fig. 5, the piston actuation force is plotted versus the deployment time for 4 different examples of freestream velocity. As expected, the required piston force increases with Mach number and $\delta_{\mathrm{SB}}$. It is worth mentioning that in the first steps of the analysis the force is dropping near to zero beginning from a positive value (Fig. 5). This can be explained by taking into consideration the mechanism's inertial loads, that has to overcome to initiate its motion, along with slim aerodynamic loads, present at this stage of deployment. In the following steps of the simulation, aerodynamic pressure becomes the principal loading condition, introducing a continue ascent, in terms of working loads for the mechanism, as the Mach number and the deflection angle increases.

During the initial material evaluation and selection study for the SB mechanism design, a list of desirable material properties was created. The working environment was defined as an extremely demanding one considering mechanical and thermal loads and high temperature corrosion phenomena, given that the mechanism should be functioning in temperatures up to $650^{\circ} \mathrm{C}$, with heavy repeating loads and instantaneously changes in loading conditions. From the initial pool of candidate material families, metallic alloys were selected due to their sufficient mechanical properties in high temperatures, desirable machinability parameters, low cost and, finally, due to well established analysis methods about their overall mechanical behavior making them handy and suitable for a conceptual mechanism design, being presented. Among metallic materials, the titanium-aluminide family of materials was compliant with the criteria that were set, and more specifically, the Ti-6Al-4V was selected as reference alloy, a well-known choice for many hypersonic applications.

The concept SB and all the actuating mechanism parts are sized under maximum calculated loads, using FEA. The analysis is performed on BETA EPILYSIS solver, calculating stresses on solid or surface elements, generated on the BETA ANSA 19.1.1 preprocessing software. The main load condition for the SB is out-of-plane bending. The SB is presented as a flat plate being modeled by shell elements (NASTRAN CQUAD4 SHELL elements). The boundary conditions used for the SB model are a support structure represented by fully constrained (in all of 6 degrees of freedom) nodes on every hinge area (a total of three areas) and a distributed pressure load, acting perpendicular to the plate (Fig. 6). In order to achieve a maximum deformation of $4 \mathrm{~mm}$ on the SB, the bump's thickness is calculated to be no less than $4.2 \mathrm{~mm}$. Considering that the selected material for the SB would be a high temperature resistance TiAl alloy with density $4.43 \mathrm{~g} / \mathrm{cm}^{3}$ (for example Ti-6Al-4V), it is expected that the total bump weight to be $0.8 \mathrm{~kg}$. Correspondingly, the other two main components of the actuating mechanism, the piston rod and the connecting rod, are modeled as solids using NASTRAN CPENTA and CHEXA elements. The main load condition for these rods is compression thus the maximum calculating stress should be a function of the cylindrical rod's diameter, in order to avoid buckling. The boundary conditions are a fixed and one side of the rod and the working compressing load one the other, calculated by the kinematic analysis, on the other side.

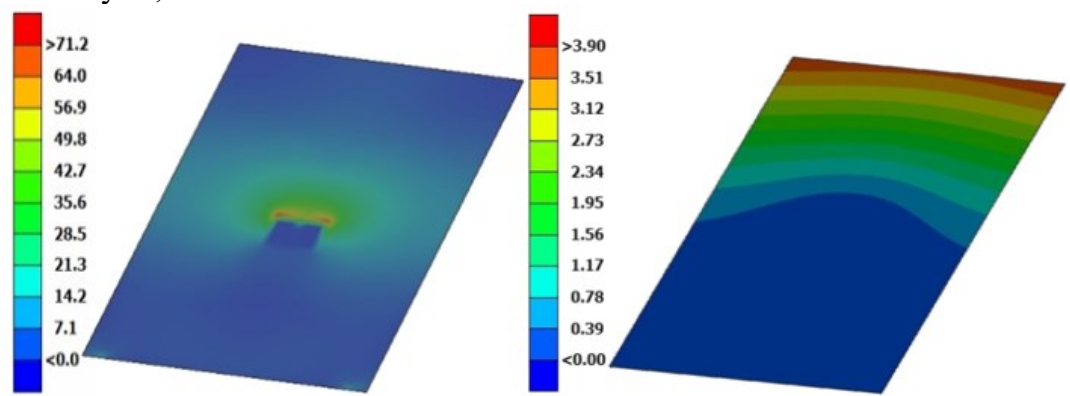

Fig. 6. Von Mises stresses [MPa] and total deformation [mm] of the plate SB at Mach 7 and $\delta_{\mathrm{SB}}=40 \mathrm{deg}$. 


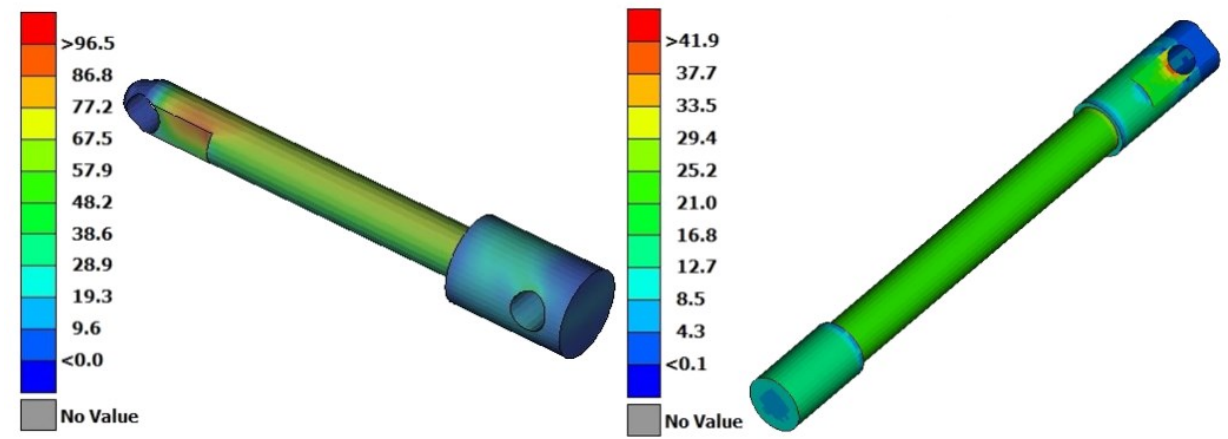

Fig. 7. Connecting and piston rod stress results $[\mathrm{MPa}]$ at Mach 7 and $\delta_{\mathrm{SB}}=40 \mathrm{deg}$.

The FEA results indicate a minimum diameter of $8 \mathrm{~mm}$ for both analyzed rods. Due to the previously presented kinematic analysis, the length of each rod is fixed, thus the calculation of a minimum diameter is sufficient for an initial weight estimation. The minimum estimated weight for the connecting rod is $0.6 \mathrm{~kg}$ and $0.8 \mathrm{~kg}$ for the actuating rod, since Ti-6Al-4V alloy is selected to also be used in these cases.

Table 1. SB Concept overview

\begin{tabular}{ll}
\hline Operating Mach range & $4-7 \mathrm{Mach}$ \\
Max. deflection angle & $40 \mathrm{deg}$ \\
Nominal deflection time & $0.1 \mathrm{sec}$ \\
Structure & Ti-6Al-4V \\
Overall mechanism weight & $2.5 \mathrm{~kg}$ \\
\hline
\end{tabular}

\section{Conclusions}

In the present research, some fundamental aspects of a novel control technique utilizing active shock bumps are examined at a conceptual level. A CFD analysis, solving the 2dimensional Euler equations, is performed in the proposed SB geometry, and is validated with theoretical results. For a total number of 20 cases, the SB is examined in 4 different velocities and 5 different device deflection angles, and the results are presented in a 3D plot, containing interpolated values for all intermediate Mach numbers and deflection angles. Moreover, a mechanism that can operate the SB system is conceptualized and analysed from a kinetic and kinematic point of view. The kinematics of the device are simple, without any singularity points, whereas the stress loads on its joints are within realistic values. Following an initial material selection investigation, it can be concluded that the proposed SB technique is feasible, both from an aerodynamics and a kinematics point of view. However, further research is required, which could be summarized in the following suggested topics:

- Detailed evaluation of the SB technique, taking into account viscosity and aerodynamic heating due to the use of the device, and 3D flow effects.

- Thorough investigation of the potential of SB technique on the control and trimming of an aerial vehicle.

- Investigation of other types of actuating motions, such as a rotational servoactuator and mechanism optimization.

- Investigation of the thermomechanical loads on the mechanism linkages.

\section{References}

1. D. P., Raymer, Aircraft Design: A Conceptual Approach, $5^{\text {th }}$ ed., American Institute of Aeronautics and Astronautics, Reston, VA, (2012) 
2. M. H., Sadraey, Aircraft design: a systems engineering approach, Wiley, Chichester, (2013)

3. M. J., Abzug, E. E., Larrabee, Airplane stability and control: a history of the technologies that made aviation possible, $2^{\text {nd }}$ ed., Cambridge University Press, Cambridge, UK ; New York, (2002)

4. U.S. Air Force, Waverider, https://www.af.mil/About-Us/FactSheets/Display/Article/104467/x-51a-waverider/, (n.d.)

5. G., Norris, Skunk Works Reveals SR-71 Successor Plan, Aviation Week \& Space Technology, http://aviationweek.com/technology/skunk-works-reveals-sr-71successor-plan, (n.d.)

6. M., Pätzold, T., Lutz, E., Krämer, S., and Wagner, Numerical optimization of finite shock control bumps, AIAA Aerospace Sciences Meeting and Exhibit, Reno, Nevada, (2006), pp. 12656-12668

7. M. Kintscher, and H. P., Monner, Structural Concept of an Adaptive Shock Control Bump Spoiler, SAE Tech. Pap. Part F129883 (2017). doi:10.4271/201701-2164

8. P., Harsha, L., Keel, A. Castrogiovanni, and R. Sherrill, X-43A Vehicle Design and Manufacture, AIAA/CIRA International Space Planes and Hypersonics Systems and Technologies Conference (2005). doi:10.2514/6.2005-3334

9. U.S. Standard atmosphere 1976, NASA-TM-X-74335, Washington, (1976)

10. J., Kirz, and R., Rudnik, DLR TAU Simulations for the Second AIAA Sonic Boom Prediction Workshop, AIAA Applied Aerodynamics Conference, AIAA AVIATION Forum, Denver, Colorado, (2017). doi:10.2514/6.2017-3253

11. J., Kirz, Surrogate Based Shape Optimization of a Low Boom Axisymmetric Body, AIAA Applied Aerodynamics Conference, AIAA AVIATION Forum, Atlanta, Georgia, (2018). doi:10.2514/6.2018-2849

12. J., Hileman, Z., Spakovszky, M., Drela, and M., Sargeant, Airframe Design for "Silent Aircraft", AIAA Aerospace Sciences Meeting and Exhibit, NV, (2007)

13. C., Bliamis, P., Panagiotou, and K. Yakinthos, Hypersonic vehicle control concept using an active shock bump technique, AIAA International Space Planes and Hypersonic Systems and Technologies Conference, Orlando, Florida, (2018)

14. Ames research staff, Equations, tables, and charts for compressible flow, NACATR-1145, (1953)

15. J. D., Anderson, Hypersonic and high-temperature gas dynamics, $2^{\text {nd }}$ ed., American Institute of Aeronautics and Astronautics, Reston, Va, (2006)

16. L. Roy, Analysis and Synthesis of Four-Bar Mechanism, (2008)

17. S. D., Castellanos, A. J., Cavaleiro, A. M. P. D., Jesus, R., Neto, \& J. L., Alves, Machinability of titanium aluminides: A review, Proc. of the Inst. of Mech. Eng., Part L: Journal of Materials: Design and Applications, 233(3), 426-451, (2019)

18. D.R., Tenney, W. B., Lisagor, \& S. C., Dixon, (1991). October 1988. Material and Structures for Hypersonic Vehicles, NASA Technical Memorandum. 